\title{
Valence bands of poly(methylmethacrylate) and photoion emission in vacuum ultraviolet region
}

\author{
N. Ueno \\ Department of Materials Science, Faculty of Engineering, Chiba University, Inage-ku, Chiba 263, Japan \\ Y. Kobayashi \\ Department of Image Science and Technology, Faculty of Engineering, Chiba University, Inage-ku, \\ Chiba 263, Japan \\ T. Sekiguchi \\ Department of Chemistry, Faculty of Science, Tokyo Institute of Technology, Meguro, Tokyo 152, Japan \\ H. Ikeura \\ Department of Chemistry, Faculty of Science, University of Tokyo, Bunkyo, Tokyo 113, Japan \\ K. Sugita \\ Department of Materials Science, Faculty of Engineering, Chiba University, Inage-ku, Chiba 263, Japan \\ K. Honma \\ Department of Chemistry, Faculty of Science, Himeji Institute of Technology, Akoh 678-12, Japan \\ K. Tanaka \\ Photon Factory, National Laboratory for High Energy Physics, Tsukuba 305, Japan
}

\author{
E. Ortí and R. Viruela \\ Department de Quimica Fisica, Facultat de Ciencies Quimiques, Universitat de Valencia, Dr. Moliner 50, \\ E-46100 Burjassot, Spain
}

(Received 1 June 1992; accepted for publication 8 August 1992)

\begin{abstract}
Photoion and photoelectron yields were measured for poly (methylmethacrylate) in the photon energy region of $8-40 \mathrm{eV}$ using synchrotron radiation. Further, the valence-band structure was investigated with ultraviolet photoelectron spectra and valence effective Hamiltonian calculations. A significant difference was observed between the photon energy dependencies of photoion and photoelectron yields. The threshold energy for photoion emission was found to be $10.5 \mathrm{eV}$, while that for photoelectron emission was $8.5 \mathrm{eV}$, indicating holes created near the valence-band top do not contribute to the ion emission. At the higher-energy region, the ion emission efficiency was found to be enhanced in the photon energy region of $17-28 \mathrm{eV}$. The difference between the threshold energies of photoion and photoelectron emission and the enhancement of the photoion emission are discussed in conjunction with the valence-band structure.
\end{abstract}

\section{INTRODUCTION}

The relaxation of electronically excited states of solids involves electron emission, photon emission, and chemicalbond scission followed by emission of ionic and neutral species, depending on the energies and nature of the excited states. When solids are excited by vacuum ultraviolet light (VUV) and soft $\mathrm{x}$ rays (SX), a deeper-lying hole localized at a definite atom can be created and it is expected to play a crucial role in the chemical-bond scission near this atom. In fact, such a decomposition was observed in various systems, especially for simple molecules ${ }^{1,2}$ and for adsorbed systems of small molecules on metal and semiconductor surfaces. ${ }^{3-5}$ Further, two holes can be produced due to Auger-type decay and they enhance the bond scission by so-called Coulomb explosion. ${ }^{1}$

Although the photochemical decomposition of polymer solids by ionizing radiation has been widely used in $x$-ray and electron beam lithography, the knowledge on the decomposition mechanism is very limited. In general, the photochemical decomposition of polymers was investigated by measuring the reduction of molecular weight, and infrared- and UV-absorption measurements. ${ }^{6,7}$ These mea- surements usually ignored the bond scission which produces only a small change of the molecular weight and/or products of short lifetime.

On the other hand, the photon energy dependence of photochemical decomposition of polymer solids was studied by measuring the mass spectra of neutral species vaporized from the polymers. ${ }^{8-10} \mathrm{~A}$ principal drawback of these investigations is that the fragments produced at the inside of the sample can diffuse to the surface followed by vaporization, and the diffusion time depends largely on the structure and size of the fragments. Further, they may bring about additional reaction to produce additional species. In fact, serious changes of the mass spectra and photoetching rate were observed depending on the irradiation time, temperature, and thickness of polymer samples. ${ }^{10,11}$ Therefore, it is difficult to obtain precise information on the photochemical decomposition of polymers from experiments that observe the neutral species.

Photochemical decomposition by ionizing photons can produce ionic fragments (photoions) which must reflect a characteristic of the decomposition. We expect that photo- 


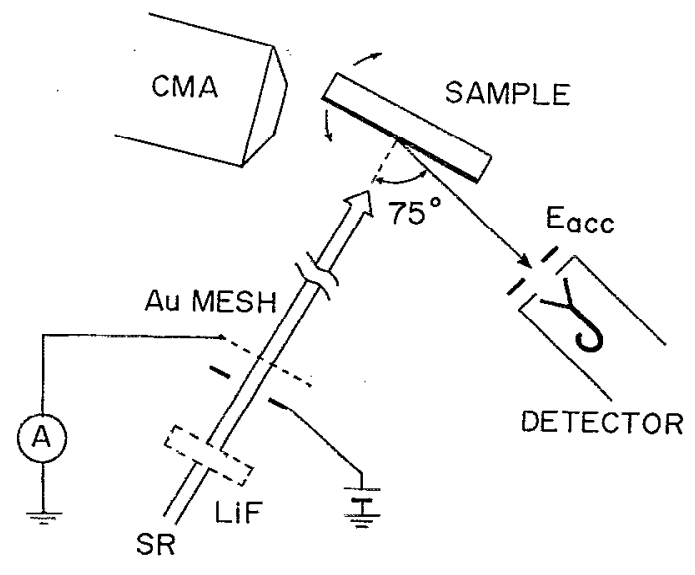

FIG. 1. Scheme for experimental setup. CMA: double-pass cylindrical mirror analyzer for photoelectron spectra. $E_{\text {acc }}$ i electrode for electron and ion collection. A LiF filter was used for the measurements with photon energies below $12 \mathrm{eV}$ to reduce the second-order light.

ions produced inside the film are neutralized easily during their diffusion or transport to the surface.

In the present investigation, we measured the photon energy dependence of total photoion yield (TIY) and total electron yield (TEY) for poly (methylmethacrylate) (PMMA) in the VUV region in order to study the VUVinduced decomposition of PMMA. Further, we investigated the valence-band structure of PMMA with ultraviolet photoelectron spectroscopy (UPS) and theoretical calculations with the valence effective Hamiltonian (VEH)

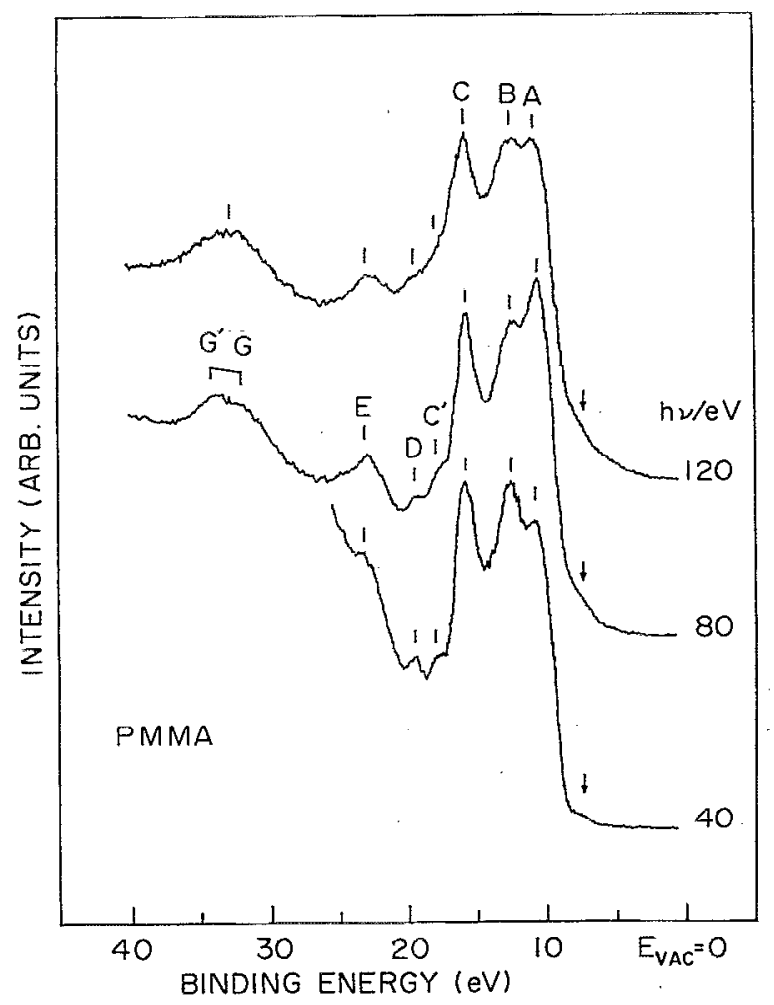

FIG. 2. Photoelectron spectra of PMMA at photon energies of 40,80 , and $120 \mathrm{eV}$. method, and discussed the difference that was observed between the photon-energy dependencies of TEY and TIY.

\section{EXPERIMENT}

We have carried out two types of experiments: (i) TIY and TEY measurements in the VUV region; and (ii) measurements of UPS spectra. These experiments were performed at the beam lines BL-12A [experiments (i) and (ii)] and BL-12C [experiment (ii)], utilizing SeyaNamioka and plane grating monochromators, respectively, at the Photon Factory of the National Laboratory for High Energy Physics. ${ }^{12}$

The intensity of incident photon flux was monitored by measuring the photoelectron yield from a gold-plated tungsten mesh which was placed between the exit slit of the monochromator and measurement chamber. The experimental setup is schematically shown in Fig. 1.

In the yield experiments, the incidence angle of photons was carefully determined in order to reduce the contribution of electrons and ions photoemitted from the inner wall of the measurement chamber by photons reflected by the sample. The reflected beam irradiated an inner wall of the beam duct between the measurement chamber and the photon-flux monitor to give little contribution to the final results.

TIY and TEY were measured by changing the polarity of electric field between the sample and the detector. The spectral resolution of TIY and TEY was dominated by the wavelength step, which is shown in each result, in the stepwise sweep of the wavelength.

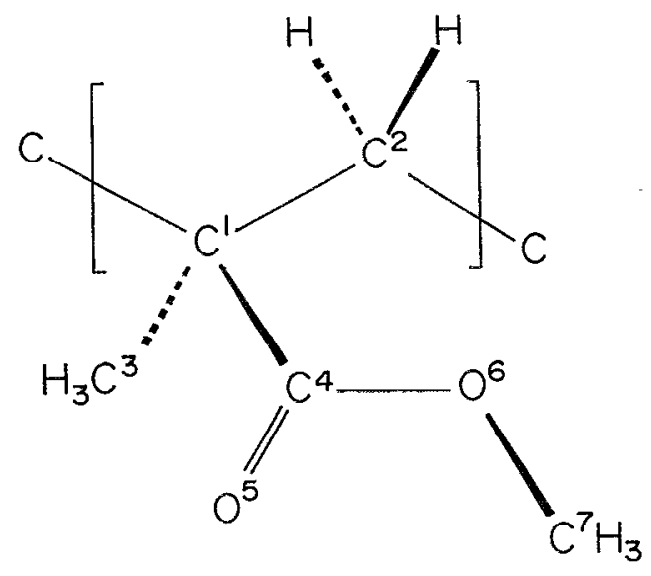

$$
\begin{aligned}
& \text { BOND LENGTH ( } \AA \text { ) BOND ANGLE (DEG) } \\
& C-C^{1}=c^{1}-C^{2}=1.54 \quad C-C^{1}-C^{2}=112 \\
& \mathrm{c}^{1}-\mathrm{C}^{3}=1.54 \quad \mathrm{c}^{1}-\mathrm{c}^{2}-\mathrm{C}=112 \\
& C^{1}-C^{4}=1.52 \quad C-C^{1}-C^{3}=108.8 \\
& c^{4}-o^{5}=1.22 \quad \text { C } C^{1}-c^{4}=108.8 \\
& c^{4}-0^{6}=1.36 \quad c^{1}-c^{4}-0^{5}=122 \\
& 0^{6}-c^{7}=1.45 \quad c^{1}-c^{4}-0^{6}=114 \\
& \mathrm{C}-\mathrm{H}=1.08 \quad \mathrm{C}^{4}-\mathrm{O}^{6}-\mathrm{C}^{7}=110
\end{aligned}
$$

FIG. 3. Schematic representation of the structural unit cell used for VEF band-structure calculations on $s$-PMMA. The atom numbering and the geometrical parameters are shown. 
TABLE I. Measured (UPS) and calculated (VEH) binding energies ( $E_{B} / \mathrm{eV}$ ) from vacuun level and VEH origins of UPS features. sh and sp denote shoulder and small peak, respectively.

\begin{tabular}{|c|c|c|c|c|c|c|}
\hline $\begin{array}{l}\text { UPS } \\
\text { features }\end{array}$ & $\begin{array}{l}\text { VEH } \\
\text { features }\end{array}$ & Origin $^{a}$ & $\mathrm{VEH}\left(E_{B}\right)^{\mathrm{b}}$ & $\operatorname{VEH}\left(E_{B}\right)^{\mathrm{c}}$ & $\operatorname{UPS}\left(E_{B}\right)$ & $\operatorname{XPS}\left(E_{B}\right)^{\mathrm{d}}$ \\
\hline Threshold & $\begin{array}{l}S \\
\mathrm{~A}_{1} \\
\mathrm{~A}_{2}\end{array}$ & $\begin{array}{l}\mathrm{O}^{5} \text { lone pairs } \\
\mathrm{O}^{6} \pi \text { lone pairs }\end{array}$ & $\begin{array}{l}10.18 \\
11.30(\mathrm{sh}) \\
12.10\end{array}$ & $\begin{array}{r}9.29 \\
10.19 \\
10.83\end{array}$ & $\begin{array}{l}8.5 \\
(9.8) \\
10.8\end{array}$ & 10.34 \\
\hline B & B & $\mathrm{O}^{6} \sigma$ lone pairs & 13.88 & 12.25 & 12.5 & 12.34 \\
\hline $\begin{array}{l}\mathrm{C} \\
\mathrm{C}^{\prime}\end{array}$ & $\begin{array}{l}\mathrm{C}_{1} \\
\mathrm{C}_{2} \\
\mathrm{C}_{3} \\
\mathrm{C}_{4}\end{array}$ & $\begin{array}{l}\mathrm{C}_{2 p}^{3}-\mathrm{H}_{1 s} \\
\mathrm{C}_{2 p}^{7}-\mathrm{H}_{1 s} \\
\mathrm{O}^{5} \text { lone pairs } \\
\mathrm{C}^{4} \mathrm{O}^{6} \mathrm{C}^{7} 2 p \sigma \text { bond }\end{array}$ & $\begin{array}{l}16.25 \\
17.41(\mathrm{sp}) \\
18.44 \\
19.67\end{array}$ & $\begin{array}{l}14.11 \\
15.08 \\
15.90 \\
16.88\end{array}$ & $\begin{array}{l}15.9 \\
17.9\end{array}$ & $\begin{array}{l}13.34(\mathrm{sh}) \\
15.90 \\
18.04\end{array}$ \\
\hline $\mathbf{D}$ & D & $\begin{array}{l}\mathrm{O}^{5} \mathrm{C}^{4} \mathrm{O}^{6} 2 s \text { antibonding and } \\
\mathrm{PE} \mathrm{C}_{25}-\mathrm{C}_{2 s} \text { antibonding }\end{array}$ & $\begin{array}{l}22.26 \\
23.53(\mathrm{sp})\end{array}$ & $\begin{array}{l}18.96 \\
19.97\end{array}$ & 19.5 & 19.59 \\
\hline E & $\begin{array}{l}E_{1} \\
E_{2}\end{array}$ & $\begin{array}{l}\mathrm{C}_{2 s}^{2} \mathrm{C}_{2 s}^{3} \\
\mathrm{C}_{2 s}^{7}\end{array}$ & $\begin{array}{l}25.60 \\
26.68\end{array}$ & $\begin{array}{l}21.63 \\
22.49\end{array}$ & 23.0 & $\begin{array}{l}21.74(\mathrm{sh}) \\
22.54\end{array}$ \\
\hline & $\mathrm{F}$ & PE $C_{25}-C_{2 s}$ bonding & 35.00 & 29.15 & & 25.14 \\
\hline $\begin{array}{l}\mathrm{G} \\
\mathrm{G}^{\prime}\end{array}$ & $\begin{array}{l}\mathrm{G}_{1} \\
\mathrm{G}_{2}\end{array}$ & $\begin{array}{l}\mathrm{O}_{2 s} \\
\mathrm{O}^{5} \mathrm{C}^{4} \mathrm{O}^{6} 2 s \text { bonding }\end{array}$ & $\begin{array}{l}37.67 \\
40.95 \\
41.87(\mathrm{sh})\end{array}$ & $\begin{array}{l}31.28 \\
33.91 \\
34.64\end{array}$ & $\begin{array}{l}32.1 \\
33.9\end{array}$ & $\begin{array}{l}31.84 \\
33.39 \\
34.54\end{array}$ \\
\hline
\end{tabular}

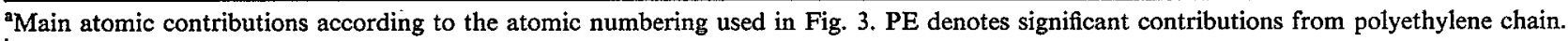
bVH binding energies calculated for an isolated chain of s-PMMA.

${ }^{\mathrm{V}} \mathrm{VEH}$ binding energies after a contraction of 1.25 of energy scale and a rigid shift of the energy scale to align the theoretical peak $\mathrm{C}_{3}$ with UPS peak $\mathrm{C}$ at $15.9 \mathrm{eV}$.

dXPS results for s-PMMA are cited from Ref. 14. The binding-energy scale is shifted by $5.54 \mathrm{eV}$ to align the XPS peak C with UPS peak C.

The UPS spectra were measured with a double-pass cylindrical mirror analyzer (CMA) (ULVAC- $\Phi, 15-$ $255 \mathrm{G})$ under the total resolution of $0.3 \mathrm{eV}(h v=40 \mathrm{eV})$ to $1 \mathrm{eV}(h v=120 \mathrm{eV})$. The binding-energy scale was calibrated by measuring the spectra of copper phthalocyanine $\left(\alpha\right.$-form crystal). ${ }^{13}$

$M_{W}$ and $M_{w} / M_{n}$ of PMMA used were $6 \times 10^{5}$ and 2.14 , respectively. The tacticity was determined by nuclear-magnetic-resonance (NMR) measurements to be $i$ (isotactic): $h$ (heterotactic): $s$ (syndiotactic) =4.1:36.9:59.0. Thin films of PMMA were spin cast on $\mathrm{Si}(100)$ wafers and gold-evaporated $\mathrm{Si}(100)$ wafers. The thickness of the PMMA films was determined so that both charging of the film and contributions from the substrate do not affect the final results. The film thickness $T$ was estimated to be $T \leqslant 100 \AA$ with a multiple interference microscope.

The typical vacuum condition of the measurement chambers was $10^{-9}-10^{-8}$ Torr throughout the experiments. All results presented here were obtained for PMMA films on $\mathrm{Si}(100)$ and at room temperature, since similar TIY and TEY results were observed for PMMA films on gold-evaporated $\mathrm{Si}(100)$ and at a sample temperature of about $80^{\circ} \mathrm{C}$.

\section{RESULTS AND DISCUSSION}

\section{A. UPS spectra}

Before presenting the TIY and TEY results, we first show examples of the angle-integrated photoelectron spectra with photon energies of 40, 80, and $120 \mathrm{eV}$ in Fig. 2. The spectra with 80 and $120 \mathrm{eV}$ photons display all features that originate in $\mathrm{C}_{25}$ and $\mathrm{O}_{25}$-derived states as well as
$\mathrm{C}_{2 p^{-}}, \mathrm{O}_{2 p^{-}}$, and $\mathbf{H}_{1 s^{-}}$-derived states. They show eight apparent features $A-G^{\prime}$. Near the right-hand-side cutoff of the spectra, a tail is seen as shown by the arrow. We tentatively ascribe this tail to electronic states produced by radiation damages, since the intensity of this tail increased with an increase in irradiation time of the photons. The ionization threshold was determined to be $8.5 \mathrm{eV}$ from the righthand-side cutoff of the photoelectron spectra. The binding energies of the UPS features are tabulated in Table I.

\section{B. Theoretical band structure}

The detailed results of the theoretical calculation of the density of states (DOS) are described elsewhere. ${ }^{14}$ Concisely, the theoretical approach employed is the VEH quantum-mechanical technique, which has been shown to provide an accurate picture of the valence electronic structure for a wide variety of polymers. ${ }^{15-21}$ The VEH method takes only into account the valence electrons and is well documented in the literature. ${ }^{15,17,22-24}$ It is parametrized to provide one-electron energies of Hartree-Fock $a b$ initio double-zeta quality. The VEH atomic potentials used in this calculation are those previously optimized for hydrogen, carbon, and oxygen. ${ }^{24,25}$ The DOS has been calculated from the electronic band structure following the methodology of Delhalle and Delhalle. ${ }^{26}$ The structural parameters (bond length and bond angle) ${ }^{27}$ used to build up the unit cell are shown in Fig. 3. It was confirmed that calculations using main-chain angles ranging from $109.5^{\circ}$ to $124^{\circ}$ introduce only slight changes in the valence-band structure. This is because the electronic states are mainly determined by the pendant groups as shown below. 


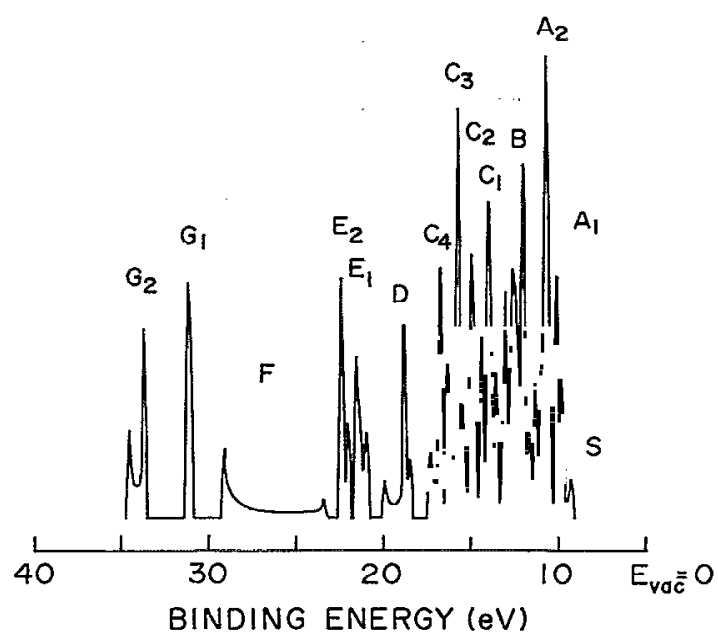

FIG. 4. VEH density of states for an isolated s-PMMA chain. The binding-energy scale of the DOS curve is shown after a contraction of 1.25 in energy scale and a rigid shift $(2.54 \mathrm{eV}$ ) (see Table I for uncollected results).

In Fig. 4, the calculated DOS for syndiotactic PMMA (s-PMMA) chain is shown. Starting from the low-bindingenergy side in Fig. 4, there is a small DOS peak $(S)$ at the top of the valence band, which is characterized by $\mathrm{C}_{2 p}$ interactions along the main chain.

A roughly triple-peak structure is presented in $A$. The most intense peak $\left(\mathrm{A}_{2}\right)$ involves mostly the lone pairs $(\pi$ contribution) of $\mathrm{O}^{6}$. At its low-binding-energy side, there is a double peak $\left(A_{1}\right)$ originating in the $O^{5}$ lone pairs.

Peak $B$ contains three main peaks. The most intense peak corresponds to $\sigma$ contributions to the lone pairs of $\mathrm{O}^{6}$. The next two peaks involve contributions from $\mathrm{C}_{2 p}-\mathrm{H}_{1 \mathrm{~s}} \sigma$ bonding and carbonyl $\pi$ bonding.

Next, we find a group of bands labeled $\mathrm{C}_{1}-\mathrm{C}_{4}$. These bands mainly imply contributions from carbon and oxygen $2 p$ orbitals and hydrogen $1 s$ orbitals. Peak $\mathrm{C}_{1}$ involves both $\mathrm{C}_{2 p}^{3}-\mathrm{H}_{1 s}$ and $\mathrm{O}_{2 p}^{6}-\mathrm{C}_{2 p}^{7}-\mathrm{H}_{1 s} \sigma$-bonding interactions. Peak $\mathrm{C}_{2}$ involves mainly $\mathrm{C}_{2 p}^{7}-\mathrm{H}_{1 s}$ bonding interactions with some contributions from $\mathrm{O}_{2 p}^{6}$ orbitals. The intense peak $\mathrm{C}_{3}$ corresponds to the lone pairs of carbonyl oxygen atoms. The lowest band gives rise to peak $\mathrm{C}_{4}$ originating from the $\mathrm{C}^{4}-\mathrm{O}^{6}-\mathrm{C}^{7} \sigma$ bonding.

The structure $\mathrm{D}$ is mainly localized on the transverse chain formed by the $\mathrm{C}^{1}$ atom and its methyl and ester substituents. The main contribution is from the $2 s$ orbitals of the $-\mathrm{C}^{4} \mathrm{O}^{5} \mathrm{O}^{6}$ - carboxylate group.

The DOS peak $\mathrm{E}_{1}$ originates mainly in $\mathrm{C}^{2}$ and $\mathrm{C}^{3} 2 s$ orbitals, while peak $\mathrm{E}_{2}$ originates in the $\mathrm{C}_{25}^{7}$ orbital. Both bands involve some $\mathrm{C}-\mathrm{H}$ bonding interactions.

The DOS feature $F$ results from the bonding interactions of the $2 s$ orbitals of the carbon atoms forming the main backbone of PMMA. It corresponds to the deep-lying $\mathrm{C}_{2 s}$ band of polyethylene. ${ }^{28}$

Peaks $G_{1}$ and $G_{2}$ are mainly built from $\mathrm{O}_{2 s}$ atomic orbitals. Peak $G_{1}$ is totally localized on oxygen atoms, while $G_{2}$ originates in the bonding interactions of oxygen

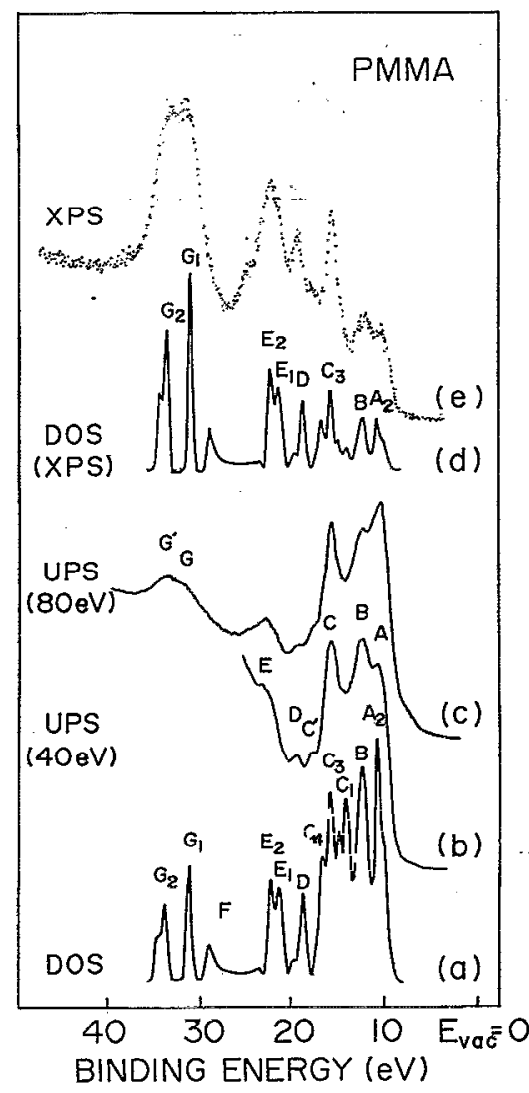

FIG. 5. Comparison of experimental and theoretical valence-band structures: (a) VEH density of states for s-PMMA after $0.7 \mathrm{eV}$ broadening; (b) and (c) UPS spectra of PMMA at photon energies of 40 and $80 \mathrm{eV}$, respectively; (d) VEH density of states for $s$-PMMA after the collection of XPS ionization cross section and $0.7 \mathrm{eV}$ broadening (sce Ref. 14); (c) high-resolution XPS spectrum for s-PMMA (see Ref. 14). The binding energies of the DOS curves (a) and (d) result after a contraction of 1.25 in energy scale and a rigid shift to align the theoretical peak $\mathrm{C}_{3}$ with experimental peak $\mathrm{C}$.

atoms with the $2 s$ orbital of the $\mathrm{C}^{4}$ atom. These results are also summarized in Table I.

\section{Comparison of UPS and calculated results}

In Fig. 5, the UPS spectra are compared with both convoluted DOS curves and a high-resolution x-ray photoelectron spectroscopy (XPS) spectrum ${ }^{14}$ measured for $s$-PMMA. In order to make a detailed comparison between the experimental and calculated results, we first note that (i) the ratio of the ionization cross sections for $s$ - and $p$-type electrons, $\sigma_{s} / \sigma_{p}$, becomes larger in XPS spectra than UPS spectra, ${ }^{29}$ and (ii) the theoretical results in general need some corrections in their binding energy scales. ${ }^{14,28} \mathrm{It}$ is seen in Fig. 5 that the UPS features show excellent agreement with the XPS features, and peaks C, D, E, and G are observed more intensely in the XPS spectrum. This indicates that peaks $\mathrm{C}, \mathrm{D}, \mathrm{E}, \mathrm{G}$, and $\mathrm{G}^{\prime}$ originate in the electronic states derived from $s$-type orbitals. On the basis of these results, we performed a detailed comparison between the UPS band structure and the calculated results. The DOS curve (a) was obtained by a Gaussian broadening of the VEH DOS in Fig. 4 with a FWHM value of $0.7 \mathrm{eV}$. 


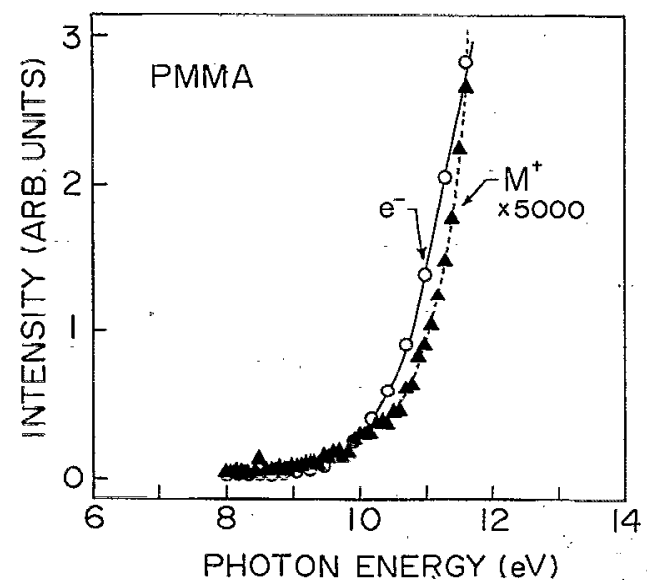

FIG. 6. Photon energy dependence of photoion and photoelectron emission from PMMA near the ionization threshold. The photon energy dependence of the incidence photon flux is included.

The DOS curve (d) was further modified by the photoionization cross-section model of Gelius ${ }^{29}$ for a simulation of the XPS spectrum. ${ }^{14}$ Further, the binding-energy scales of these DOS curves were contracted by 1.25 and shifted rigidly to align the theoretical peak $\mathrm{C}_{3}$ with the UPS peak $\mathrm{C}$ at $15.9 \mathrm{eV}$. As seen in Fig. 5, an excellent agreement was obtained between the experimental and theoretical results. On the basis of this excellent agreement, the origins of the UPS features are summarized in Table I.

\section{Photoion and photoelectron yields}

Figure 6 displays raw results of the photon energy dependencies of the photoion and photoelectron intensities around ionization threshold, where the photon energy dependence of the intensity of the incidence is not collected. In these measurements, ${ }^{9} \mathrm{LiF}$ single-crystal filter was used to reduce the second-order light. The clear observation of photoions indicates that the photochemical decomposition of PMMA by ionizing radiation produces ionic species. It is further seen that there is a clear difference between the threshold energies for photoion and photoelectron emission. In order to determine the threshold energies for electron and ion emission, cube root plots of TIY and TEY are shown in Fig. 7, after dividing the raw results by the photon energy dependence of the photoelectron intensity from the gold mesh. The cube root plot was used successfully in determining the threshold energy of polyethylene. ${ }^{30}$ From these plots, we obtained a linear relation between the cube root of TEY and TIY and photon energy, and determined the threshold energy to be $8.5 \mathrm{eV}$ for photoelectron emission and $10.5 \mathrm{eV}$ for photoion emission. This threshold energy for TEY agrees excellently with that determined from the photoelectron spectra, indicating that the collection of the raw data by the photoelectron intensity from the gold mesh is reasonable in determining the threshold energies.

It is notable that there is a remarkable difference of 2 $\mathrm{eV}$ between the threshold energies for TIY and TEY. This indicates that the ionization from valence states at $0-2 \mathrm{eV}$

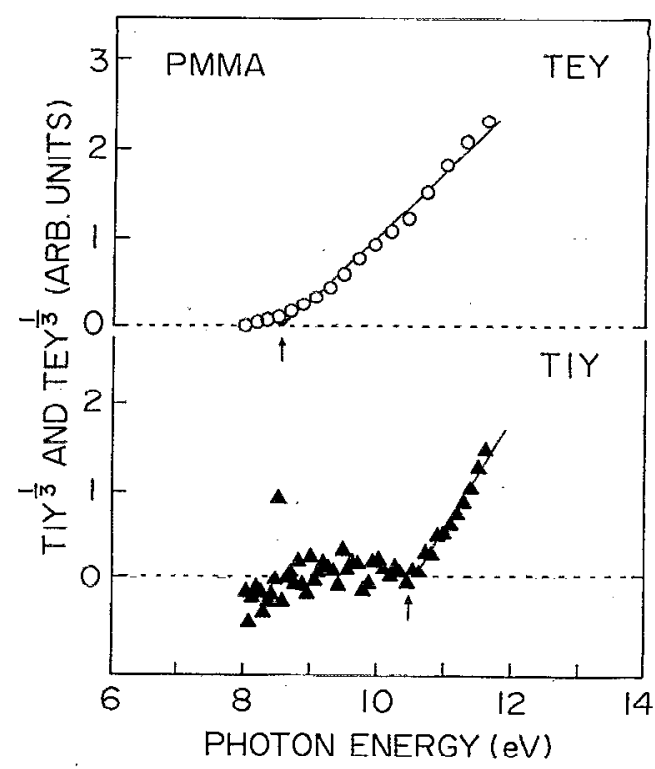

FIG. 7. Cube root plots for TIY and TEY. Threshold energies for TIY and TEY are indicated by arrows.

below the valence-band top does not produce bond scission which creates ionic species. From the results of the valence-band analysis, the ion-emissive molecular decomposition starts by an ionization from $\pi$ lone pairs at $\mathrm{O}^{6}$, while it does not take place by creating holes at the states originating $\mathrm{C}_{2 p}$ interactions along the chain and $\mathrm{O}^{5}$ lone pairs.

Figure 8 displays the photon energy dependence of TEY and TIY in the photon energy range of $12-40 \mathrm{eV}$, together with the absorption coefficient obtained by Ritsko et $a l .{ }^{31}$ Here, TIY and TEY are obtained by the correction of the raw data using both the reflectivity of PMMA ${ }^{31}$ and the incidence intensity after a correction of the photon energy dependence of photoelectron yield of gold. ${ }^{32}$ Figure 8 shows that both TIY and TEY have a maximum near 16 $\mathrm{eV}$ which coincides well with the absorption maximum. Further, it shows that there is remarkable difference between TIY and TEY in the photon energy range $18-26 \mathrm{eV}$.

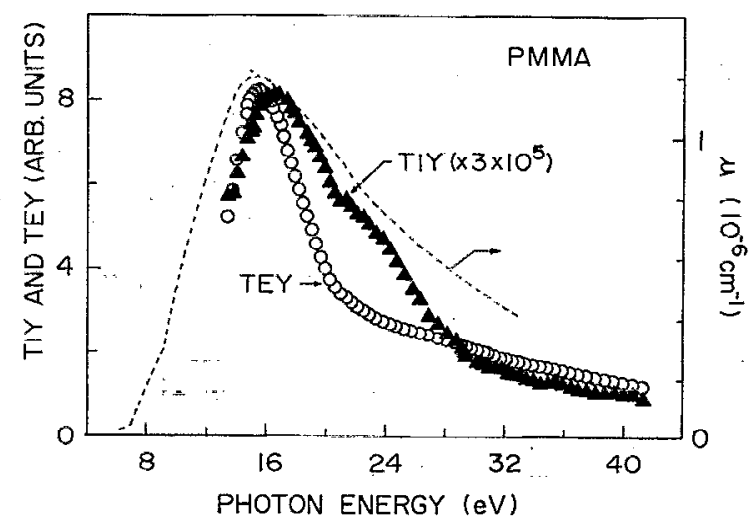

FIG. 8. Photon energy dependence of TIY and TEY for PMMA in the photon energy region of $12-40 \mathrm{eV}$. The absorption coefficient (see Ref. 31 ) is shown by the broken curve. 


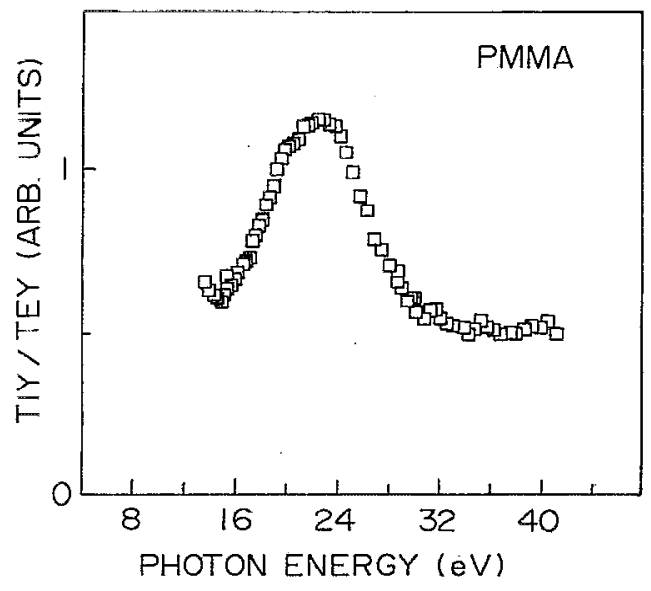

FIG. 9. Photon energy dependence of ion emission efficiency (TIY/ TEY).

In Fig. 9, we show the ratio TIY/TEY which represents the photon energy dependence of the photoion emission efficiency, and this curve is free from an ambiguity which is in general introduced into the yield curves by the correction of the data with the reflectivity and the estimated intensity of photon flux. The result clearly shows a broad peak at about $23 \mathrm{eV}$. Since the ion emission should be only observed by photochemical decomposition of PMMA, the results indicate that the decomposition is accelerated by about two times when PMMA is excited by about $23 \mathrm{eV}$ photons.

The absorption of VUV by solids in general occurs by various electronic excitations such as ionization and creation of super excited states ${ }^{33}$ (high-energy excitons). The latter involves many electronic transitions of similar transition energy from occupied states to unoccupied states. Further, these excited states can autoionize to produce free electrons. Therefore, it is very difficult to discuss the definite origins of the features in the yield curves in this energy region. However, we note here that the energy giving the peak structure in TIY/TEY corresponds closely to the binding energy of the $\mathrm{C}_{25}$-derived bands (feature $\mathrm{E}$ in Fig. 5 and Table I). We tentatively ascribe the increase of the photoion emission efficiency to electron excitations from the $\mathrm{C}_{25}$-derived states. In passing, the unoccupied states of PMMA can be considered to be located at more than 5.79 $\mathrm{eV}$ above the valence-band top, since the first absorption band is observed at a photon energy of $5.79 \mathrm{eV}{ }^{31}$ Thus the photons of energies greater than $20.29 \mathrm{eV}$ may create $\mathrm{C}_{2 s}$ holes, and above $23 \mathrm{eV}$ direct ionization can take place. However, the clear peak structure in TIY/TEY at about 23 $\mathrm{eV}$ suggests that this peak originates in the excitations of the $\mathrm{C}_{2 s}$ electron to the resonance states, since the ionization cross section generally decreases gradually with increase in photon energy. As a result, PMMA decomposes more effectively to give ionic species by exciting electrons of $\mathrm{C}_{25}$ derived states than those of $\mathrm{O}_{2 s}$-derived states.

For more detailed consideration, we must know the structure of photoemitted ions. Measurements of the pho- ton energy dependence of time-of-flight mass spectra of the photoemitted ions are now in progress using pulsed synchrotron radiation.

\section{ACKNOWLEDGMENTS}

The authors are very grateful to the staff of the Photon Factory at the National Laboratory for High Energy Physics for their help in various ways. This work was partly supported by the DGICYT Project PS88-0112 of the "Ministerio de Educacion y Ciencia" of Spain.

${ }^{1}$ See, for example, T. A. Carlson, in Desorption Induced by Electronic Transitions, edited by N. H. Tolk, M. M. Traum, J. C. Scully, and T. C. Madey (Springer, Berlin, 1983), p. 169.

${ }^{2}$ W. Eberhardt, T. K. Sham, R. Carr, S. Krummacher, M. Strongin, S. L. Weng, and D. Wesner, Phys. Rev. Lett. 50, 1038 (1983).

${ }^{3}$ P. J. Feibelman, Surf. Sci. 102, L51 (1981).

${ }^{4}$ M. L. Knotck and P. J. Fcibelman, Phys. Rev. B 18, 6531 (1978).

${ }^{5}$ K. Tanaka, H. Ikeura, N. Ueno, Y. Kobayashi, K. Obi, T. Sekiguchi, and K. Honma, in Synchrotron Radiation and Dynamic Phenomena, edited by A. Beswick (American Institute of Physics, New York, 1992), p. 332.

${ }^{6}$ A. R. Shultz, P. Frank, B. F. Griffing, and A. L. Young, J. Polym. Sci. Polym. Phys. Ed. 23, 1749 (1985).

'J. O. Choi, J. A. Moore, J. C. Corelli, J. P. Silverman, and H. Bakhru, J. Vac. Sci. Technol. B 6, 2286 (1988).

${ }^{8}$ K. Mochiji, T. Kimura, H. Obayashi, and M. Yanagihara, Appl. Phys. Lett. 45, 251 (1984).

${ }^{9}$ H. Yamada, M. Hori, S. Morita, and S. Hațtori, J. Electrochem. Soc. Solid-State Sci. Tech. 135, 966 (1988).

${ }^{10}$ N. Ueno, T. Mitsuhata, K. Sugita, and K. Tanaka, Polymers in Microlithography, ACS Symp. Ser. Vol. 412 (American Chemical Society, Washington, DC, 1989), Chap. 26.

${ }^{11}$ N. Ueno and K. Sugita, Jpn. J. Appl. Phys. Part 1 25, 1455 (1986).

${ }^{12}$ T. Namioka, Photochcm. Photobiol. 44, 245 (1986).

${ }^{13}$ S. X. Chen, K. Seki, H. Inokuchi, Z. R. Shi, and R. Y. Qian, Bull. Chem. Soc. Jpn. 56, 2565 (1983).

${ }^{14}$ E. Orti, R. Viruela, J. L. Brédas, and J. J. Pireaux, Polymer-Solid Interfaces (in press).

${ }^{15}$ S. P. Kowalczyk, S. Stafström, J. L. Brédas, W. R. Salaneck, and J. L. Jordan-Sweet, Phys. Rev. B 41, 1645 (1990).

${ }^{16}$ M. Chtaib, J. Ghijsen, J. J. Pireaux, R. Caudano, R. L. Johnson, E. Ortí, and J. L. Brédas, Phys. Rev. B 44, 10815 (1991).

${ }^{17}$ J. L. Brédas, R. R. Chance, R. Silbey, G. Nicolas, and Ph. Durand, J. Chem. Phys. 77, 371 (1982).

${ }^{18}$ J. L. Brédas and W. R. Salaneck, J. Chem. Phys. 85, 2219 (1986).

${ }^{19}$ E. Ortí, S. Stafström, and J. L. Brédas, Chem. Phys. Lett. 164, 240 (1989).

${ }^{20}$ E. Ortí, J. L. Brédas, J. J. Pireaux, and N. Ishihara, J. Electron. Spectrosc. Relat. Phenom. 52, 551 (1990).

${ }^{21}$ R. Lazzaroni, N. Sato, W. R. Salaneck, M. C. Dos Santos, J. L. Brédas, B. Tooze, and D. T. Clark, Chem. Phys. Lett. 175, 175 (1990).

${ }^{22}$ G. Nicolas and Ph. Durand, J. Chem. Phys. 72, 453 (1980).

${ }^{23}$ J. M. André, L. A. Burke, J. Delhalle, G. Nicolas, and Ph. Durand, Int. J. Quantum Chcm. Symp. 13, 283 (1979).

${ }^{24} \mathrm{~J}$. L. Brédas, R. R. Chance, R. Silbey, G. Nicolas, and Ph. Durand, J. Chem. Phys. 25, 255 (1981).

${ }^{25}$ B. Themans, J. M. André, and J. L. Brédas, Mol. Cryst. Liq. Cryst. 118, 121 (1985).

${ }^{26}$ J. Delhalle and S. Delhalle, Int. J. Quantum Chem. 11, 349 (1977).

${ }^{27}$ M. Vacatello and P. J. Flory, Macromolecules 19, 405 (1986).

${ }^{28}$ N. Ueno, K. Seki, N. Sato, H. Fujimoto, T. Kuramochi, K. Sugita, and H. Inokuchi, Phys. Rev. B 41, 1176 (1990).

${ }^{29}$ U. Gelius, in Electron Spectroscopy, edited by D. A. Shirley (NorthHolland, Amsterdam, 1972), p. 311.

${ }^{30}$ S. Hashimoto, K. Seki, N. Sato, and H. Inokuchi, J. Chem. Phys. 76, 163 (1982).

${ }^{31}$ J. J. Ritsko, L. J. Brillson, R. W. Bigelow, and T. J. Fabish, J. Chem. Phys. 69, 3931 (1978).

${ }^{32}$ R. B. Cairns and J. A. R. Samson, J. Opt. Soc. Am. 56, 1568 (1966).

${ }^{33}$ R. L. Platzman, Radiat. Res. 17, 419 (1962). 